\section{A Multiband Quasi-Yagi Type Antenna}

Sung-Jung Wu, Cheng-Hung Kang, Keng-Hsien Chen, and Jenn-Hwan Tarng

\begin{abstract}
A new design of multiband quasi-Yagi type antenna for 700 MHz band, GSM900, DCS1800, GPS, and Bluetooth/WLAN applications is presented. In contrast to conventional quasi-Yagi antenna design, our proposed antenna realizes the multiband performance by interaction between the section extended ground and the derived driver element which is a branch of driver dipole element. The parametric studies of the proposed antenna are discussed to explore the antenna operating mechanism. The performances of the antenna are demonstrated along with measured and simulated results.
\end{abstract}

Index Terms-700 MHz band, antenna, multiband antenna, quasi-Yagi antenna.

\section{INTRODUCTION}

With the growing demand for wireless communication, wireless applications such as $2 \mathrm{G} / 3 \mathrm{G}$ cellular phone, global positioning system (GPS), wireless local area network (WLAN), and Bluetooth have been developed at a fast pace during the past decade. On July 31, 2007, the Federal Communications Commission (FCC) revised the $700 \mathrm{MHz}$ band plan and service rules to establish a nationwide, interoperable public safety broadband network for the benefit of state and local public safety consumer [1]. The $700 \mathrm{MHz}$ band, ranging from 698 to 806 $\mathrm{MHz}$, is expected to be used for commercial multimedia broadcasting, digital television, and local public safety network in regulated channels.

Several studies have reported applications and technologies of the multiband antenna design, including the internal quad-band handset antenna of compact size [2], single and double layer multiband planar inverted-F antenna (PIFA) [3], multi-resonant monopole antenna [4], and multiband multiple ring monopole antenna [5]. Different from the multiband antenna, broadband antennas have been designed for high speed transmission rates and high capacity broadband services. The broadband sleeve monopole antenna [6] and metal-plate monopole antenna [7] were designed for DTVs and portable multimedia players, respectively.

The quasi-Yagi antenna has been widely used in wireless communication because of its broadband characteristics and good radiation performance [8], [9]. This antenna has various transitions, is inexpensive, has a low profile, is easy to implement, and can readily be integrated with other circuit components. For example, the quasi-Yagi antennas were designed with microstrip-to-CPS transitions in order to connect to other components [10], [11] and a $100 \mathrm{GHz}$ quasi-Yagi antenna was achieved using a silicon process [12].

In this paper, we present a new design for the multiband quasi-Yagi antenna for the $700 \mathrm{MHz}$ (698-806 MHz), GSM900 (880-960 MHz), DCS1800 (1720-1880 MHz), GPS (1575 MHz), and Bluetooth/WLAN (2400-2484 MHz) applications. The proposed antenna shows the three section operating bands (measured return loss $>7$ )

Manuscript received May 01, 2009; revised October 07, 2009. Current version published February 03, 2010. This work was supported by the National Science Council, R.O.C., under Grants NSC 98-2221-E-009-051 and NSC 98-2219-E009-001.

The authors are with Department of Communication Engineering, National Chiao Tung University, Hsin-Chu, Taiwan, R.O.C.(e-mail: sungjungwu.cm96g@nctu.edu.tw).

Digital Object Identifier 10.1109/TAP.2010.2041522

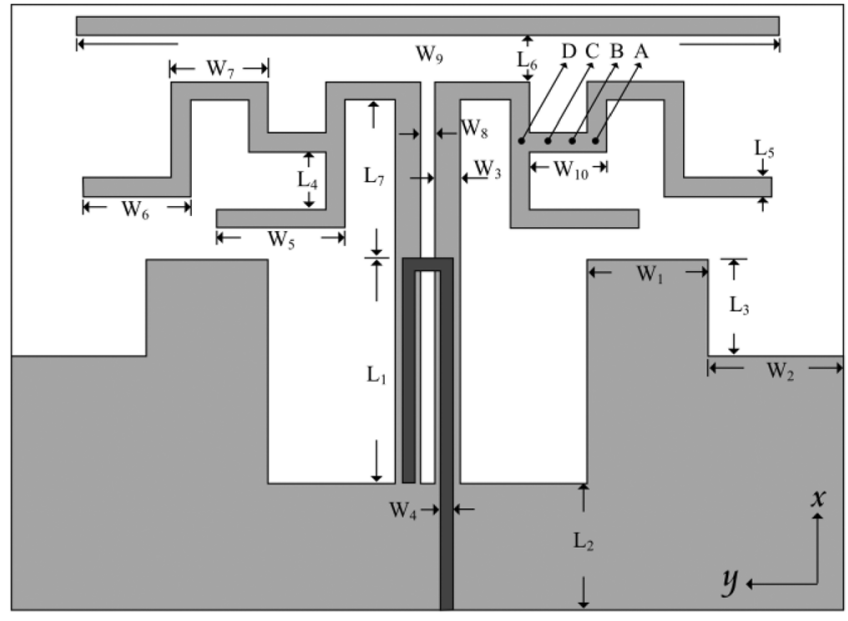

(a)

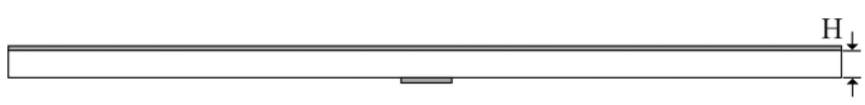

(b)

Fig. 1. Configuration of the proposed antenna. (a) Top view. (b) Cross-sectional view.

which cover the above-mentioned applications and provide acceptable absolute gains ( $0-4.4 \mathrm{dBi})$. The initial design of the proposed antenna begins with a conventional quasi-Yagi antenna. Furthermore, the interaction between the section extended ground plane and the branch of driver dipole element is employed for multiband performance.

The organization of this paper is followed. In Section II, we present the geometry and design concept of the proposed antenna as well as important parameters for the design process. The measured and simulated radiation patterns are given in Section III. Finally, we present the conclusions in Section IV.

\section{ANTENNA CONFIGURATION AND DESIGN THEORY}

Fig. 1 shows the proposed multiband quasi-Yagi type antenna. The initial design begins with a conventional quasi-Yagi antenna. The antenna consists of a microstrip-to-CPS transition, a driver dipole element, a parasitic director element, a derived driver element, and a truncated ground plane as the reflector element of the proposed antenna. The derived driver element is a branch of the driver dipole element. The driver dipole element is wound to reduce the antenna size. The parasitic director element on the top plane simultaneously directs the wave propagation toward the end-fire direction, and acts as an impedance matching element. The antenna was fabricated on a $1 \mathrm{~mm}$ FR4 substrate of size of $130 \mathrm{~mm} \times 95 \mathrm{~mm}$. The final antenna parameters are optimized by using the commercial electromagnetic solver HFSS 9.2: $\mathrm{W}_{1}=\mathrm{W}_{2}=21, \mathrm{~W}_{3}=4, \mathrm{~W}_{4}=\mathrm{W}_{8}=2, \mathrm{~W}_{5}=20, \mathrm{~W}_{6}=$ $17, \mathrm{~W}_{7}=15, \mathrm{~W}_{9}=110, \mathrm{~W}_{10}=12, \mathrm{~L}_{1}=55, \mathrm{~L}_{2}=20, \mathrm{~L}_{3}=$ $15, \mathrm{~L}_{4}=9, \mathrm{~L}_{5}=3, \mathrm{~L}_{6}=7, \mathrm{~L}_{7}=28, \mathrm{H}=1$, where all units are in $\mathrm{mm}$.

The measured return loss agrees with the simulated one as shown in Fig. 2. There are three section operating bandwidths, which are on the $10 \mathrm{~dB}$ return loss condition. It is clear that the derived driver element not only provides impedance matching around the GPS and DCS1800 bands but also improves the performance for nearby the $700 \mathrm{MHz}$ and GSM900 bands. Here, the microstrip-to-CPS transition forms a folding 


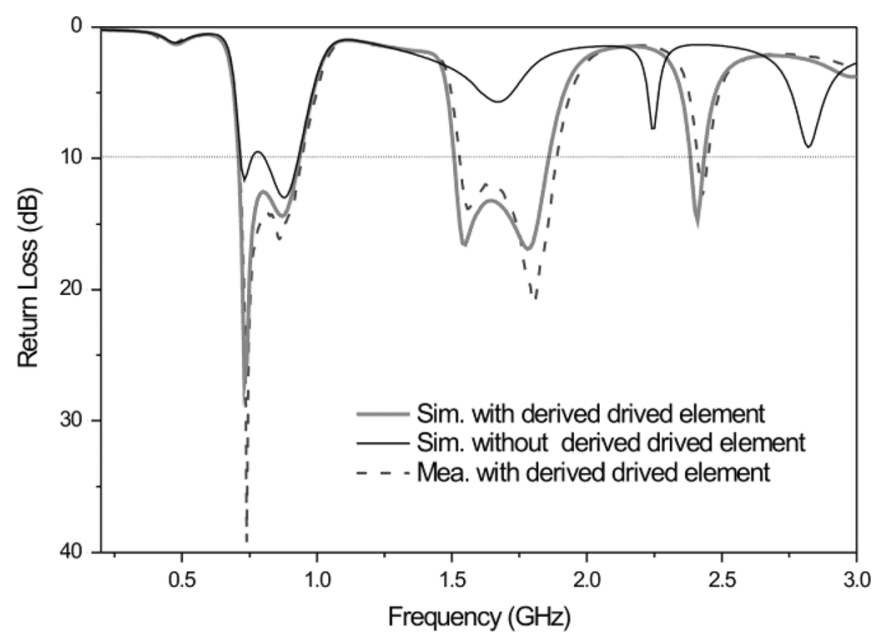

Fig. 2. Measured and simulated return loss of proposed antenna.

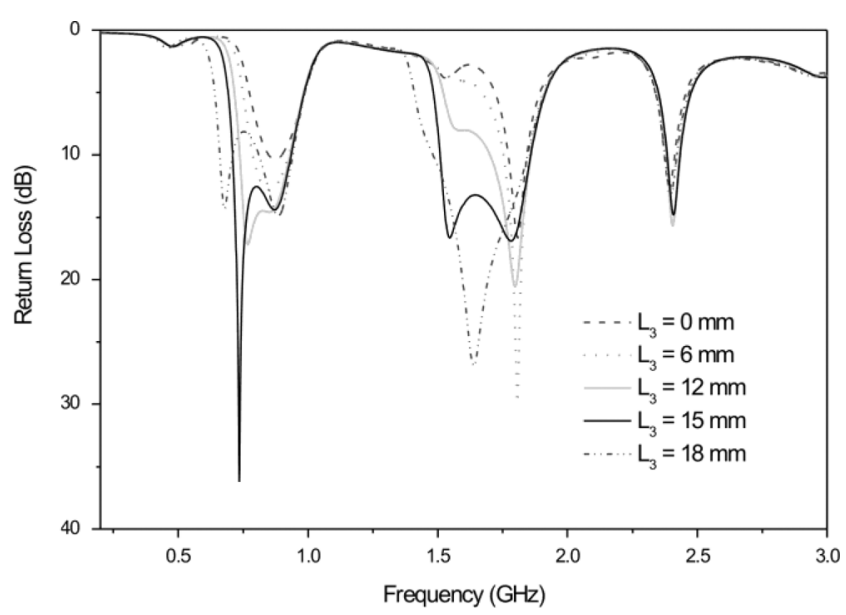

Fig. 3. Simulated return loss of various lengths of the section extended ground.

microstrip line and exhibits broadband impedance transforming properties. The transition is designed to excite the odd-mode at the CPS line by providing $180^{\circ}$ phase delay and is capable of transforming an unbalanced input signal to a balanced signal at the driver dipole element. In order to maximize the antenna impedance bandwidth, the optimum length of $L_{1}$ is around one-eighth the length of the lowest operating frequency.

To further improve the front-to-back ratio and the impedance matching of the proposed antenna, a part of the ground plane is extended toward the radiator. Fig. 3 shows the simulated return loss of various lengths of the section extended ground. With this arrangement, the bandwidth of each band becomes large when $\mathrm{L}_{3}$ changes from 0 to $15 \mathrm{~mm}$. However, bandwidth of $700 \mathrm{MHz}$ and GSM900 bands decrease as $\mathrm{L}_{3}=18 \mathrm{~mm}$.

Fig. 4 shows the simulated return loss for various derived driver element positions. The derived driver element connects to different locations on the driver dipole element, i.e., Points A, B, C, and D. For the GPS and DCS1800 bands, the bandwidth changes at various locations. The derived driver element not only influences the lowest operating frequency slightly but also strongly relates to the bandwidth of the GPS and DCS bands.

Fig. 5 shows the simulated return loss for various distances between the driver dipole element and the parasitic director element. At the

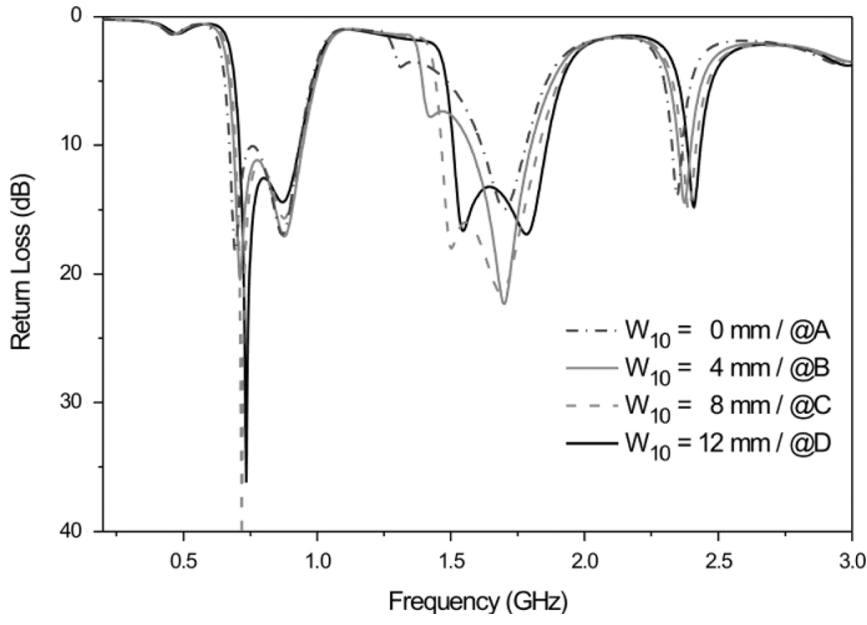

Fig. 4. Simulated return loss for various derived driver element positions.

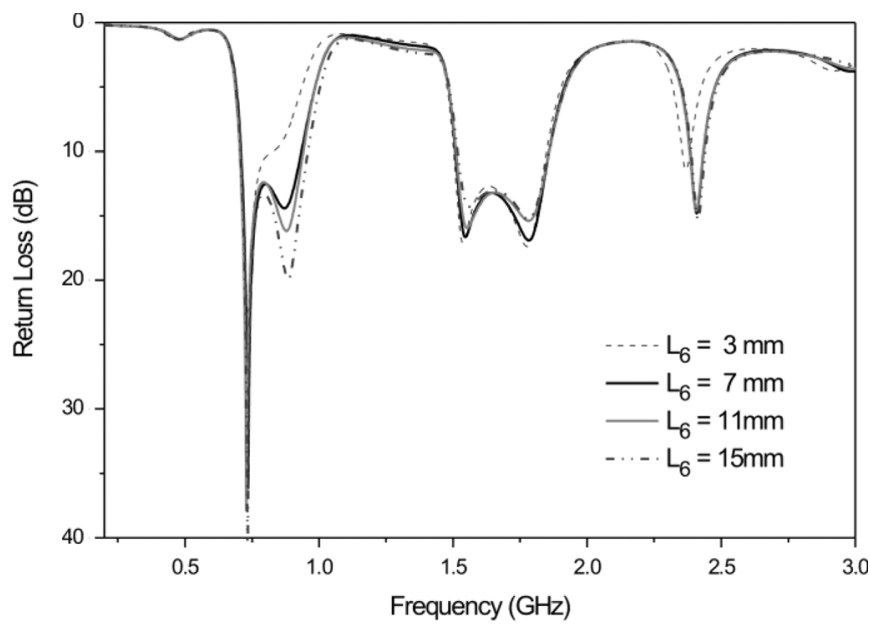

Fig. 5. Simulated return loss for various distances between the driver dipole element and the parasitic director element.

$700 \mathrm{MHz}$ and GSM900 bands, the distance becomes shorter, which strengthens the coupling and helps the wave propagate along the end-fire direction, ensuring a good end-fire pattern. However, the coupling also affects the wide impedance bandwidth.

Although the bandwidths of the proposed antenna do not accurately cover the operating frequency of the wireless standards on $10 \mathrm{~dB}$ returnloss condition, the parameter studies are still useful for providing the design guideline. According to the Fig. 2 to 5, the lowest operating frequency is strongly related to the length of the driver dipole element. Furthermore, the bandwidths of each band are related to the capacitive interaction between the derived driver element and extended ground plane. Thus, by adjusting the position of the derived driver element and the extended ground plane, the bandwidths of the proposed antenna may satisfy bandwidth of wireless standards.

\section{RADIATED PATTERN}

The antenna radiation patterns are measured in a $7.0 \mathrm{~m} \times 3.6 \mathrm{~m} \times 3.0$ $\mathrm{m}$ anechoic chamber with an Agilent E362B network analyzer and the NSI2000 far-field measurement software. The xy- and the yz-plane radiation for each band are illustrated in Fig. 6. The agreement between the simulations and measurements is fairly good in most of the results. The discrepancies between simulated and measured cross-polarization 

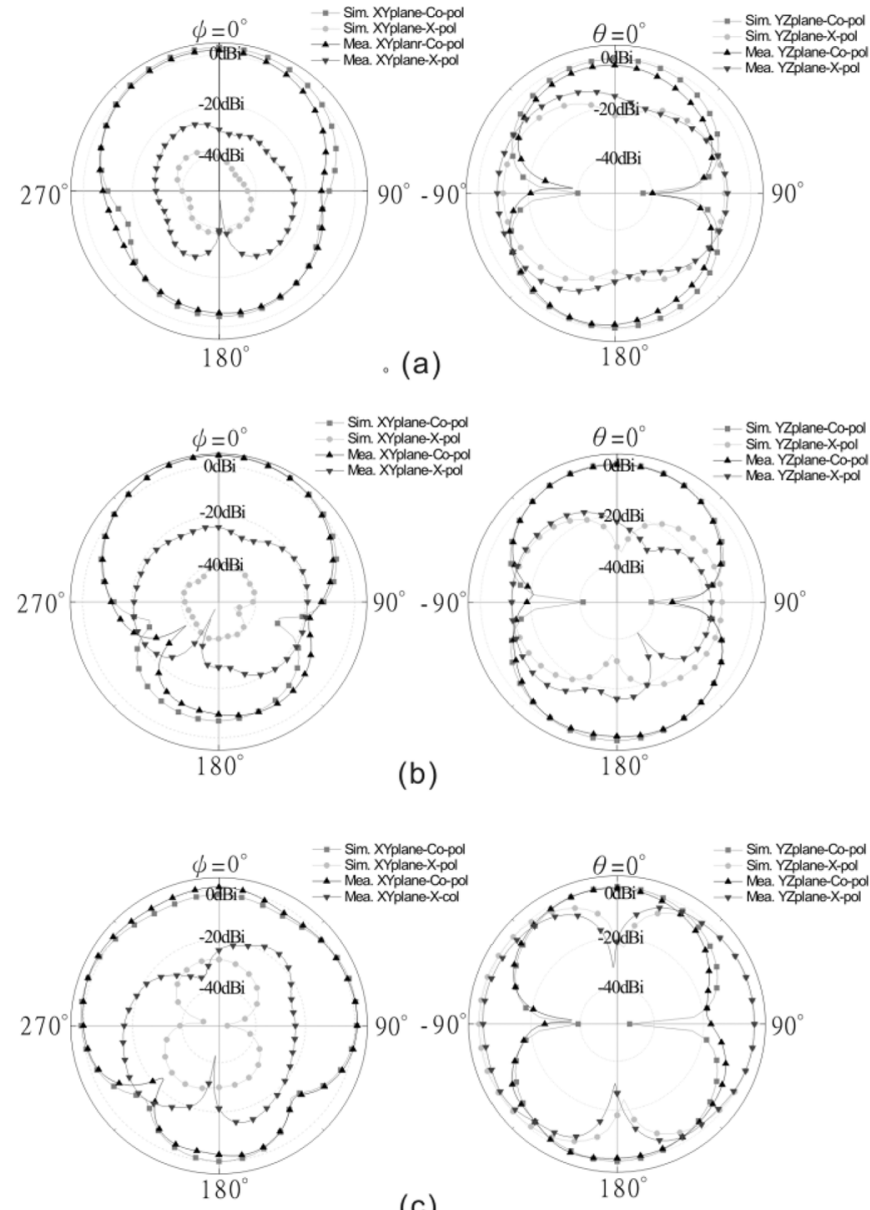

(c)
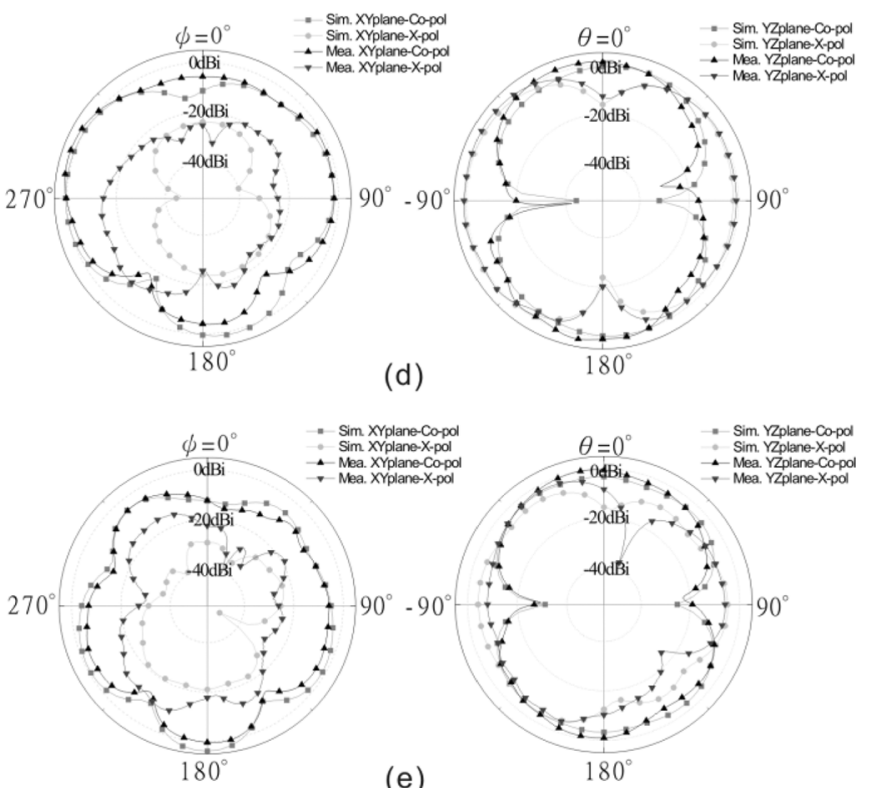

Fig. 6. Simulated and Measured radiation patterns (a) at $750 \mathrm{MHz}$. (b) at 900 MHz. (c) at $1575 \mathrm{MHz}$. (d) at $1800 \mathrm{MHz}$. (e) at $2400 \mathrm{MHz}$. (Unit: dBi)

level in xy-plane can be mostly attributed to the measured uncertainty such as the connecting coaxial cable and the absorber used in measured arrangement.
TABLE I

List OF THE MEASURED BAND CHARACTERISTICS

\begin{tabular}{|c|c|c|c|}
\hline Band & Bandwidth $^{* 1}$ & Gain $(\mathrm{dBi})$ & $\begin{array}{c}\text { Front-to-Back } \\
\text { ratio }\end{array}$ \\
\hline $700 \mathrm{MHz}$ & 700 937MHz & $3.13 \mathrm{dBi}$ & $7.3 \mathrm{~dB}$ \\
\hline $900 \mathrm{MHz}$ & $28.96 \%$ & $4.4 \mathrm{dBi}$ & $11.2 \mathrm{~dB}$ \\
\hline $1575 \mathrm{MHz}$ & $1508 \sim 1860 \mathrm{MHz}$ & $1.1 \mathrm{dBi}$ & \multirow{2}{*}{ N/A } \\
\hline $1800 \mathrm{MHz}$ & $20.9 \%$ & $1.1 \mathrm{dBi}$ & \\
\hline $2400 \mathrm{MHz}$ & $\begin{array}{c}2382 \sim 2435 \mathrm{MHz} \\
2.2 \%\end{array}$ & $0.3 \mathrm{dBi}$ & N/A \\
\hline
\end{tabular}

Note 1: The 10dB return loss condition is considered.

Referring to Fig. 6(a) and (b), the co-polarized patterns in xy-plane demonstrate end-fire radiation patterns. The measured cross-polarized gains are around $20 \mathrm{~dB}$ lower than the co-polarized one in the xy-plane. It is not a surprising result because the operating mechanism of the proposed antenna has the characteristics of the quasi-Yagi radiated. Referring from Fig. 6(c) to (d), the cross-polarized level becomes larger than the one in Fig. 6(a) and (b). The radiated patterns do not present the end-fired forms because the operating mechanism of the proposed antenna is realized by capacitive interaction between the extended ground plane and the derived driver element. Referring to Fig. 6(e), the maximum gain in the xy-plane is at $\psi=180^{\circ}$.

The measured gain and bandwidths of the proposed antenna are shown in Table I. Three measured section bandwidths on the 10 $\mathrm{dB}$ return loss condition are $700-937 \mathrm{MHz}, 1508-1860 \mathrm{MHz}$, and 2382-2435 MHz, respectively. If the $7 \mathrm{~dB}$ return loss condition is adjusted, the operating bandwidths of the proposed antenna cover the bandwidth of $700 \mathrm{MHz}$ band, GSM900, DCS1800, GPS, and Bluetooth/WLAN standards. The measured maximum gains are 0.3-4.4 dBi within operating bands. This is in good agreement with the simulated results.

\section{CONCLUSION}

In this paper, a new multiband quasi-Yagi type antenna has been proposed for $700 \mathrm{MHz}$ band, GSM900, DCS1800, GPS, and Bluetooth/ WLAN applications. The antenna configuration and design methodology have been discussed. The set of parametric studies of the proposed antenna provides brief guidelines for the antenna designer. Simultaneously, the measured return loss and radiation patterns agree with the simulated ones. The proposed antenna is expected to find applications in wireless communication.

\section{REFERENCES}

[1] [Online]. Available: http://www.fcc.gov/pshs/public-safety-spectrum/ 700-MHz/

[2] Y.-X. Guo, I. Ang, and M. Y. W. Chia, "Compact internal multiband antennas for mobile handsets," IEEE Antennas Wireless Propag. Lett., vol. 2, pp. 143-146, 2003.

[3] B. Sanz-Izquierdo, J. C. Batchelor, R. J. Langley, and M. I. Sobhy, "Single and double layer planar multiband PIFAs," IEEE Trans. Antennas Propag., vol. 54, no. 5, pp. 1416-1422, May 2006.

[4] S. Hong, W. Kim, H. Park, S. Kahng, and J. Choi, "Design of an internal multiresonant monopole antenna for GSM900/DCS1800/USPCS/S-DMB operation," IEEE Trans. Antennas Propag., vol. 56, no. 5, pp. 1437-1443, May 2008.

[5] C. T. P. Song, P. S. Hall, and H. Ghafouri-Shiraz, "Multiband multiple ring monopole antennas," IEEE Trans. Antennas Propag., vol. 51, no. 4, pp. 722-729, Apr. 2003.

[6] H.-D. Chen, "Compact broadband microstrip-line-fed sleeve monopole antenna for DTV application and ground plane effect," IEEE Antennas Wireless Propag. Lett., vol. 7, pp. 497-500, 2008. 
[7] Y.-S. Yu, D.-H. Seo, S.-G. Jeon, and J.-H. Choi, "Design of an internal DTV antenna for portable multimedia player," in Proc. Asia-Pacific Microwave Conf., Dec. 12-15, 2006, pp. 1601-1603.

[8] N. Kaneda, W. R. Deal, Q. Yongxi, R. Waterhouse, and T. Itoh, "A broadband planar quasi-Yagi antenna," IEEE Trans. Antennas Propag., vol. 50, no. 8, pp. 1158-1160, Aug. 2002.

[9] S.-Y. Chen and P. Hsu, "Broadband microstrip-fed modified quasiYagi antenna," in IEEE/ACES Int. Conf. on Wireless Commun. and Appl. Comput. Electromagn., Apr. 3-7, 2005, pp. 208-211.

[10] D.-S. Woo, Y.-G. Kim, K. W. Kim, and Y.-K. Cho, "A simplified design of quasi-Yagi antennas using the new microstrip-to-CPS transitions," in Proc. IEEE Antennas and Propag. Society Int. Symp., Jun. 9-15, 2007, pp. 781-784.

[11] R.-C. Hua, C.-W. Wang, and T.-G. Ma, "A planar quasi-Yagi antenna with a new microstrip-to-CPS balun by artificial transmission lines," in Proc. IEEE Antennas and Propag. Society Int. Symp., Jun. 9-15, 2007, pp. 2305-2308.

[12] M. Sun and Y. P. Zhang, "100-GHz quasi-Yagi antenna in silicon technology," IEEE Electron Device Lett., vol. 28, no. 5, pp. 455-457, May 2007.

\section{A Novel Wideband and Compact Microstrip Grid Array Antenna}

Xing Chen, Guosheng Wang, and Kama Huang

\begin{abstract}
A wideband and compact microstrip grid array antenna is presented. The antenna is printed on a dielectric substrate, backed by a metal board, and directly fed from a $50 \Omega$ coaxial cable. It adopts elliptical radiation elements to enhance its impedance and gain bandwidths, and sinusoid lines to reduce its size. Structural parameters of a proposed antenna with 7 radiation elements were optimized by a parallel genetic algorithm (GA) on a cluster system. A prototype antenna was fabricated and tested. Results of simulation and measurement agree well and show the antenna exhibits encouraging properties, e.g., $S_{11}<-10 \mathrm{~dB}$ bandwidth of $25 \%$ and a $3 \mathrm{~dB}$ gain-drop bandwidth of $16.3 \%$, both of which are much wider than that of conventional grid array antennas, as well as a maximum gain of approximately $13.7 \mathrm{dBi}$ with an area-reduction factor of $47 \%$.
\end{abstract}

Index Terms-Compact, microstrip grid array antenna, wideband.

\section{INTRODUCTION}

The grid array antenna [1]-[11] is a low-profile flat and linear or circular polarization antenna. This antenna possesses advantages such as high gain, narrow beam, and low side lobes, but has not received much attention and found popular applications since it was first presented by Kraus[1]. Literatures investigating it are limited, e.g., Conti et al. [2] reported a microstrip version of the array, and H. Nakano [3]-[11] analyzed its radiation characteristics and introduced some extensions.

A grid array antenna is composed of many grid cells and backed by a conducting ground plane. Grid cells are formed by two kinds of lines. One acts as radiation elements, and another acts as transmission lines

Manuscript received July 17, 2008; revised March 22, 2009. First published December 04, 2009; current version published February 03, 2010. This work was supported by a grant from the National High Technology Research and Development Program of China (863 Program, No. 2007AA01Z279).

The authors are with the College of Electronic and Information Engineering, Sichuan University, Chengdu 610064, China (e-mail: xingcsc@ yahoo.com.cn). Digital Object Identifier 10.1109/TAP.2009.2037769 that provide a phase delay of $360^{\circ}$ at resonance to ensure the in-phase excitation of all the radiation elements. A grid array antenna may be categorized as a wire grid array antenna [1], [4]-[6], [8], [9], [11] or a microstrip grid array antenna [2], [3], [7], [10], [12] by that its grid cells are formed by metal wires or microstrip lines. In comparison with the former, the latter is easier to be fabricated because it allows the use of simple, low cost, and accurate microstrip fabrication techniques [2].

To make a grid array antenna compact, [6] uses meander lines rather than straight lines to construct the grid cells, and so that reduces the size of the antenna at a degree of $38 \%$, but the gain of the antenna is also dropped by approximately $2 \mathrm{~dB}$.

There are still some problems existing in the grid array antenna. Firstly, the input impedance of the grid array antenna, as revealed in [3], [7], sometimes is much higher than $50 \Omega$, which means an impedance transformer is necessary if the antenna is fed from a commonly used $50 \Omega$ coaxial line. Secondly, the frequency bandwidth of the grid array antenna is narrow. For example, the grid array antenna in [6] has VSWR $<2$ bandwidth of only $2.6 \%$ and a $3 \mathrm{~dB}$ gain-drop bandwidth of less than $7 \%$; the microstrip grid array antenna designed in [7] has VSWR $<2$ bandwidth of approximately $13 \%$. To the knowledge of the authors, it is the largest impedance bandwidth reported in previous literatures; in [4], [8] and [9], several grid array antennas are presented, but their $3 \mathrm{~dB}$ gain-drop bandwidth are only approximately $3.3 \%, 9 \%$, and $5.6 \%$, respectively. In our previous work [12], the genetic algorithm (GA) was employed for optimizing a microstrip grid array antenna, but the antenna's bandwidth is only $4.5 \%$.

Today, antennas with properties of small-size, low-cost, high gain and wide frequency band are desired in the wireless communication and other applications. This communication presents a new microstrip grid array antenna, which is designed to be directly fed from a $50 \Omega$ coaxial line without an impedance transformer. To enhance its frequency bandwidth and make it compact, it adopts elliptical radiation elements and sinusoid transmission lines to form its grid cells.

Traditionally, engineers work with their intuition, exact or approximate analysis, and simulation to determine antenna's structures and parameters. The method of designing antennas is mainly by hand, so is time- and labor-intensive, and unlikely produce truly optimal results. In recent years, when the high speed computer became available, more and more engineers employ optimization algorithms in the antenna design as a powerful computer aided design technology, which is able to speed the antenna design and allow for greater design complexity. Hence, this work use the GA in conjunction with the finite different time domain (FDTD) method to optimize structural parameters of the proposed antenna on a cluster system for high radiation gain, wide frequency bandwidth and large area-reduction.

Section II introduces the configuration of the new antenna. The antenna optimization based on the parallel GA is presented in Section III. Simulated and measured properties of the designed antenna are given in Section IV. Conclusions are stated in Section V.

\section{ANTENNA CONFIGURATION}

Fig. 1 illustrates a top view and a side view of the proposed microstrip grid array antenna, whose grid array is printed on a dielectric substrate of relative permittivity $\varepsilon_{r}$ and thickness $h_{1}$, and backed by a metal board. Grid cells of the antenna are composed of $Y$-directed elliptical radiation elements and $X$-directed sinusoid transmission lines. In comparison with conventional rectangular microstrip lines or straight metal wires used as radiation elements in previous literatures [6], [7], [9], [10], elliptical elements are able to enhance the frequency bandwidth of the antenna due to their smooth and broad configuration. 